\title{
Nut consumption is inversely associated with both cancer and total mortality in a Mediterranean population: prospective results from the Moli-sani study
}

\author{
Marialaura Bonaccio $^{1,2 *}$, Augusto Di Castelnuovo ${ }^{1}$, Amalia De Curtis $^{1}$, Simona Costanzo ${ }^{1}$, \\ Francesca Bracone†, Mariarosaria Persichillo ${ }^{1}$, Maria Benedetta Donati ${ }^{1}$, Giovanni de Gaetano ${ }^{1}$, and Licia \\ Iacoviello ${ }^{1}$ on behalf of the Moli-sani Project investigators $\ddagger$ \\ ${ }^{1}$ Department of Epidemiology and Prevention, IRCCS Istituto Neurologico Mediterraneo, Neuromed, 86077 Pozzilli, Isernia, Italy \\ ${ }^{2}$ Fellow of the Fondazione Umberto Veronesi, Milan, Italy
}

(Submitted 23 January 2015 - Final revision received 4 May 2015 - Accepted 4 June 2015)

\section{Abstract}

Nut intake has been associated with reduced inflammatory status and lower risk of CVD and mortality. The aim of this study was to examine the relationship between nut consumption and mortality and the role of inflammation. We conducted a population-based prospective investigation on 19386 subjects enrolled in the Moli-sani study. Food intake was recorded by the Italian version of the European Project Investigation into Cancer and Nutrition FFQ. C-reactive protein, leucocyte and platelet counts and the neutrophil to lymphocyte ratio were used as biomarkers of low-grade inflammation. Hazard ratios (HR) were calculated using multivariable Cox proportional hazard models. During a median follow-up of 4.3 years, 334 all-cause deaths occurred. As compared with subjects who never ate nuts, rare intake ( $\leq 2$ times/ month) was inversely associated with mortality (multivariable $H R=0.68 ; 0.54,0 \cdot 87$ ). At intake $\geq 8$ times/month, a greater protection was observed ( $\mathrm{HR}=0.53 ; 0.32,0.90)$. Nut intake ( $v$. no intake) conveyed a higher protection to individuals poorly adhering to the Mediterranean diet (MD). A significant reduction in cancer deaths ( $\mathrm{HR}=0.64 ; 0.44,0.94)$ was also observed, whereas the impact on CVD deaths was limited to an inverse, but not significant, trend. Biomarkers of low-grade inflammation were reduced in nut consumers but did not account for the association with mortality. In conclusion, nut intake was associated with reduced cancer and total mortality. The protection was stronger in individuals with lower adherence to MD, whereas it was similar in high-risk groups (diabetics, obese, smokers or those with metabolic syndrome), as compared with low-risk subjects. Inflammation did not explain the observed relationship.

Key words: Nuts: Mortality: Inflammation: Cancer: CVD: Stroke

A Mediterranean diet (MD) has been associated with reduced risk for major chronic diseases ${ }^{(1,2)}$ such as CVD, cancer, diabetes and neurodegenerative diseases; its beneficial effects have been partially ascribed to its high content of PUFA, antioxidants and fibres that exhibit an anti-inflammatory action ${ }^{(3)}$. Nuts represent an important component of the MD pattern and are rich in unsaturated fatty acids, minerals (magnesium, potassium and calcium) and other bioactive compounds ${ }^{(4)}$. Epidemiological evidence has linked nut intake to reduced inflammatory status ${ }^{(5)}$ or lower risk of $\mathrm{CVD}^{(6-8)}$. Limited evidence also suggests an inverse association with cancer risk attributable to the presence of anti-carcinogenic compounds ${ }^{(9)}$. Benefits of nut intake on cardiovascular health have been ascribed to the direct effect of nuts on oxidation, inflammation and vascular reactivity ${ }^{(10)}$. In addition, possible mechanisms that are able to explain the CVD advantages of nuts may be found in the documented positive effects on blood pressure $^{(11,12)}$, blood lipids ${ }^{(13,14)}$, diabetes ${ }^{(15,16)}$ and metabolic syndrome ${ }^{(17)}$. Yet the possible role of inflammation as a mediator of the association between nut consumption and total mortality has been poorly explored so far ${ }^{(18-21)}$.

The purpose of this study was to address the relationship between nut intake and total and cause-specific mortality in a large Mediterranean cohort. Furthermore, our study investigated a possible role of inflammation ${ }^{(22)}$ in explaining the association and likely advantages of nut intake in population groups sharing common risk factors for both CVD and cancer, with particular focus on subjects with obesity, diabetes, metabolic syndrome, smokers or those with poor adherence to an MD.

$\dagger$ Epicomed Research Srl, Campobasso, Italy

\$ Moli-sani study investigators are listed in the Appendix.

Abbreviations: CRP, C-reactive protein; MD, Mediterranean diet; NLR, neutrophil to lymphocyte ratio.

* Corresponding author: M. Bonaccio, fax + 39 0865927575, email marialaura.bonaccio@neuromed.it 


\section{Methods}

\section{Study population}

Data presented here are from the Moli-sani study, which is a large population-based cohort study that recruited 24325 subjects from the general population of the Molise region, a central-southern area of Italy ${ }^{(23)}$. Individuals were enrolled from March 2005 to April 2010 and were followed up for mortality for a median of 4.3 years (interquartile range: $3 \cdot 5-5 \cdot 3$ years, 84302 person/years).

After excluding individuals with prevalent CVD ( $n$ 1320), cancer ( $n$ 781) or missing data for both diseases ( $n$ 543), unreliable medical ( $n$ 235) or dietary questionnaires ( $n$ 955), subjects lost to follow-up ( $n$ 44) or with incomplete personal data ( $n$ 408), those reporting extremely low or high values for total energy intake $(<3347 \cdot 2 \mathrm{~kJ} / \mathrm{d}(<800 \mathrm{kcal} / \mathrm{d})$ in men and $2092 \mathrm{~kJ} / \mathrm{d}(500 \mathrm{kcal} / \mathrm{d})$ in women or $>16736 \mathrm{~kJ} / \mathrm{d}(>4000 \mathrm{kcal} / \mathrm{d})$ in men and $14644 \mathrm{~kJ} / \mathrm{d}(3500 \mathrm{kcal} / \mathrm{d})$ in women) and individuals with missing information on blood pressure ( $n$ 186), lipid profile ( $n$ 373) or diabetes ( $n$ 229), a total of 19386 subjects were included in the analysis. Mortality was recorded until December 2011. Overall mortality and cause-specific mortality were assessed by the Italian mortality registry (ReNCaM registry), validated by Italian death certificates (ISTAT form) and coded according to the International Classification of Diseases (ICD-9). Cardiovascular deaths were defined when the underlying cause of death had an ICD-9 code of 390-459 or 745-747, and for cancer deaths an ICD-9 code of 140-208. A critical evaluation of the diagnosis was performed, by analysing hospital medical records for hospital deaths and for other deceased patients if previously hospitalised during the follow-up. The process of ascertainment of death causes was conducted by qualified personnel blinded to the present analyses.

The Moli-sani study was approved by the Ethics Committee of the Catholic University of Rome, Italy. All participants signed an informed consent.

\section{Dietary information}

Food intake during the year before enrolment was ascertained by the validated Italian version of the 'European Project Investigation into Cancer and Nutrition' (EPIC) FFQ ${ }^{(24,25)}$, which includes 188 food items, classified into forty-five predefined food groups on the basis of similar nutrient characteristics or culinary usage.

The EPIC questionnaire has a specific question about nut intake, including the frequency of consumption of walnuts, hazelnuts, almonds and peanuts. Frequency was categorised as never, rare ( $\leq 2$ times/month), $3-7$ or $\geq 8$ times/month.

Adherence to the MD was evaluated by the Mediterranean diet score (MDS) developed by Trichopoulou et al. ${ }^{(26)}$. The scoring was based on the intake of the following nine items: vegetables, legumes, fruit and nuts, dairy products, cereals, meat and meat products, fish, alcohol and monounsaturated/ saturated fats. For most items, consumption above the study population median received 1 point; all other intakes received 0 points. Consumption below the median of dairy products, meat and meat products received 1 point. Medians were sex-specific. For ethanol, men who consumed $10-50 \mathrm{~g} / \mathrm{d}$ and women who consumed $5-25 \mathrm{~g} / \mathrm{d}$ received 1 point; otherwise, the score was 0 . The possible scores thus ranged between 0 and 9 , the latter reflecting the maximal adherence to MD. In the present study, nut intake was not included in the MDS in order to control for dietary habits. Adherence to the MD was also categorised as poor (0-3), average (4-5) and good (>5). Daily energy intake $(\mathrm{kcal} / \mathrm{d})$ was divided into quintiles.

\section{Laboratory analyses}

All blood samples were obtained from participants who had fasted overnight and had refrained from smoking for at least $6 \mathrm{~h}$. Serum lipids and blood glucose were assayed by enzymatic reaction methods using an automatic analyser (ILab 350; Instrumentation Laboratory). LDL-cholesterol was calculated according to Friedewald ${ }^{(27)}$.

High-sensitivity (hs) C-reactive protein (CRP) was measured in fresh serum samples within $3 \mathrm{~h}$ of collection by a particleenhanced immunoturbidimetric assay (IL-Coagulation-Systems ACL9000; Instrumentation Laboratory). Quality control for hs-CRP was maintained using in-house serum pool and internal laboratory standard at $1.5 \mathrm{mg} / \mathrm{l}$; inter-day CV of hs-CRP were 5.5 and $4.2 \%$, respectively.

Hemocromocytometric analysis was performed by cell count (Coulter HMX, Beckman Coulter; Instrumentation Laboratory). Neutrophil (granulocyte) to lymphocyte ratio (NLR) was calculated as a marker of low-grade inflammation ${ }^{(22,28)}$

\section{Assessment of risk factors}

BMI was calculated as $\mathrm{kg} / \mathrm{m}^{2}$ and then categorised into three levels as normal $(\leq 25)$, overweight $(>25$ and $<30)$ or obese $(\geq 30)$. Hypertension was defined as systolic blood pressure $\geq 140 \mathrm{~mm} \mathrm{Hg}$ or diastolic blood pressure $\geq 90 \mathrm{~mm} \mathrm{Hg}$ or treatment for hypertension. Hypercholesterolaemia was defined as total cholesterol $\geq 240 \mathrm{mg} / \mathrm{dl}(6.2 \mathrm{mmol} / \mathrm{l})$ or the use of specific medication. Diabetes was defined as blood glucose $\geq 126 \mathrm{mg} / \mathrm{dl}$ or the use of specific pharmacological treatment. Metabolic syndrome was defined according to Adult Treatment Panel III criteria ${ }^{(29)}$, based on at least three of these criteria: elevated waist circumference $(>102 \mathrm{~cm}$ in men, $>88 \mathrm{~cm}$ in women); elevated TAG (triglycerides) $(>150 \mathrm{mg} / \mathrm{dl}$ ) or drug treatment for elevated TAG; reduced HDL-cholesterol $<40 \mathrm{mg} /$ $\mathrm{dl}(1.03 \mathrm{mmol} / \mathrm{l})$ in men and $<50 \mathrm{mg} / \mathrm{dl}(1.3 \mathrm{mmol} / \mathrm{l})$ in women or drug treatment for reduced HDL-cholesterol, elevated blood pressure ( $>130 \mathrm{~mm} \mathrm{Hg}$ systolic blood pressure or $>85 \mathrm{~mm} \mathrm{Hg}$ diastolic blood pressure) or anti-hypertensive drug treatment in a patient with a history of hypertension; and elevated fasting glucose $(>100 \mathrm{mg} / \mathrm{dl})$ or drug treatment for elevated glucose. Subjects were classified as never-smokers, current smokers or ex-smokers (quitting from at least 1 year).

Leisure-time physical activity was assessed by a structured questionnaire (questions on walking, gardening and sport participation) ${ }^{(30)}$, and expressed as daily energy expenditure in metabolic equivalent task-hours (MET/d) and then categorised as below $(\leq 2 \cdot 27)$ or above the median $(>2 \cdot 27)$.

Educational level as an indicator of socio-economic status was considered as secondary school or lower and high school or higher. 


\section{Statistical analysis}

ANOVA for continuous or categorical variables was used to identify variables associated with the frequency of nut consumption and included socio-demographic variables (age, sex, smoking habit, educational level and leisure-time physical activity), BMI, hypertension, systolic and diastolic blood pressure, hypercholesterolaemia, diabetes, blood glucose, adherence to the MD and total energy intake. In addition, associations with biomarkers of low-grade inflammation (CRP, platelet and leucocyte counts or NLR) and lipid profile (HDL-cholesterol, LDL-cholesterol, total cholesterol and TAG) were tested. Associations with $P$ value $<0 \cdot 10$ were used in the multivariable model. However, hypercholesterolaemia and diabetes were not included in the multivariable model, as these factors are likely to be in the causal pathway of mortality protection by nuts.

Crude, age/sex-adjusted or multivariable hazard ratios (HR) with corresponding $95 \%$ confidence intervals were calculated using the Cox proportional hazard model considering subjects in the lowest category of nut consumption as the reference group. The multivariable model was controlled for energy intake, leisure-time physical activity, smoking, educational level, BMI and the MDS not including nut consumption. An additional multivariable model was further controlled for inflammatory biomarkers to test their role as possible mediators of the effect. Sensitivity analyses were undertaken to estimate the impact of nut intake within subgroups at different CVD risk. Appropriate interaction terms were added to the models to test for differences of the effect. In cause-specific and sensitivity analyses, nut intake was compared with the no-intake group.

The data analysis was generated using the SAS/STAT software, version 9.1 .3 of the SAS System for Windows ${ }^{\circledR} 2009$. SAS Institute Inc. and SAS are registered trademarks of SAS Institute Inc.

\section{Results}

Baseline characteristics of the population according to the frequency of nut intake are reported in Table 1. Compared with subjects with no nut consumption, those with the highest nut consumption had lower prevalence of diabetes and lower blood glucose levels, higher total cholesterol, LDL-cholesterol and HDL-cholesterol levels and higher energy intake.

During a median follow-up of 4.3 years, 334 overall deaths occurred, among which 104 CVD and 124 cancer deaths could be identified. Compared with those reporting no nut intake, a reduction in mortality was observed at increased consumption of nuts ( $P$ for trend $=0.0010$ ), with a protection of $47 \%$ (10-68\%) at highest intake (Table 2, model 1); these values likely reflect a plateau-like effect.

Cause-specific risks of death are reported in Table 3. As compared with those who never ate nuts, subjects consuming nuts showed a lower risk of overall mortality of $34 \%(17,47 \%)$ and reduced cancer death of $36 \%$ (6, 56\%); CVD mortality exhibited a similar, but not significant, inverse trend $(\mathrm{HR}=0.87$; $0.57,1.32$ ). When CVD deaths were limited to CHD, a nonsignificant trend of protection of $26 \%$ was found (Table 3 ). Stroke deaths were not associated with any nut intake (Table 3).
Subgroup analyses are reported in Table 4. The protection against mortality of nut intake was more evident in subjects with poor adherence to MD $(\mathrm{HR}=0.47 ; 0.31,0.71)$ as compared with those with greater adherence $(P$ for interaction $=0.022$; Table 4). Among all other subgroups, the effects of nuts on mortality were similar across different categories of consumption (Table 4).

The relationship between nut intake and biomarkers of lowgrade inflammation is reported in Table 1 . More frequent nut consumption was associated with lower levels of CRP, platelet count and the NLR. No association was found with leucocyte count.

The association between mortality and nut intake was not modified by the inclusion of CRP, platelet count and NLR (Table 2, model 2; Table 3, model 2), or by the inclusion of lipid profile (HDL- and LDL-cholesterol; data not shown).

\section{Discussion}

Results from this large prospective study show that nut intake is associated with a reduced risk of all-cause death in a general Italian population that is apparently free, at enrolment, from CVD and cancer.

As compared with subjects who did not eat nuts, participants consuming nuts $\geq 8$ times/month showed a reduced risk ( $47 \%$ ) of dying from any cause after controlling for potential confounders, including other dietary components of the MD. In addition, those eating nuts more rarely ( $\leq 2$ times/month) exhibited a lower risk of mortality of $32 \%$. When analyses were restricted to the two main groups - that is, nut consumers $v$. no consumers - we found a protection of $34 \%$ for those consuming nuts.

\section{Cause-specific risks of death}

An effort was made to address the association of nut intake with specific causes of death. Our results show that nut intake was inversely and significantly associated with reduced risk of cancer death, in agreement with a recent investigation suggesting a beneficial effect of nuts, even at modest intakes, in lowering cancer deaths in two large, independent cohorts of nurses and other male health care professionals, from the $\mathrm{USA}^{(18)}$. Further studies will be needed to identify possible biological mechanisms explaining the observed inverse relationship; so far, limited evidence supports benefits of nut consumption on some types of cancer, such as colorectal cancer ${ }^{(31)}$ or endometrial cancer in women ${ }^{(32)}$, but the consumption of nuts has often been reported in association with seeds and legumes ${ }^{(33)}$. In addition, in previous studies, the anticarcinogenic advantages of nuts appeared only in women, whereas poor effects have been documented in men ${ }^{(31,33)}$. Unfortunately, we were not able to address cancer or sexspecific analyses because of lack of a sufficient number of cases. It is noteworthy that our findings are also in agreement with results of an analysis conducted within the framework of the PREDIMED study, the very first large intervention trial on $\mathrm{MD}^{(8,19)}$ : that analysis ${ }^{(19)}$ revealed that increased frequency of nut consumption was associated with a $40 \%$ reduced risk of 
Table 1. Main characteristics of the study population according to the frequency of nut intake

Mean values and standard deviation (SD) for continuous variables (age, biochemical parameters, biomarkers of inflammation, systolic/diastolic BP and energy intake); number and percentages for categorical variables

\begin{tabular}{|c|c|c|c|c|c|c|c|c|c|}
\hline \multirow[b]{3}{*}{ Number of subjects $(n, \%)$} & \multicolumn{8}{|c|}{ Frequency of nut intake (times/month) } & \multirow{3}{*}{$\begin{array}{c}P \\
-\end{array}$} \\
\hline & \multicolumn{2}{|c|}{ Never } & \multicolumn{2}{|c|}{$\leq 2$} & \multicolumn{2}{|c|}{$3-7$} & \multicolumn{2}{|c|}{$\geq 8$} & \\
\hline & 4271 & $22 \cdot 0$ & 12341 & $63 \cdot 7$ & 1292 & $6 \cdot 7$ & 1482 & $7 \cdot 6$ & \\
\hline Age (years, mean and SD) & $55 \cdot 6$ & $12 \cdot 4$ & $54 \cdot 1$ & $11 \cdot 1$ & $53 \cdot 7$ & $10 \cdot 5$ & $55 \cdot 5$ & $10 \cdot 9$ & $<0.0001$ \\
\hline $\operatorname{Sex}(n, \%)$ & & & & & & & & & $<0.0001$ \\
\hline Women & 2570 & $60 \cdot 2$ & 6569 & $53 \cdot 2$ & 623 & $48 \cdot 2$ & 747 & $50 \cdot 4$ & \\
\hline Men & 1701 & $39 \cdot 8$ & 5772 & $46 \cdot 8$ & 669 & $51 \cdot 8$ & 735 & $49 \cdot 6$ & \\
\hline Education (n, \%)* & & & & & & & & & $<0.0001$ \\
\hline Secondary school or lower & 2411 & $56 \cdot 5$ & 6109 & $49 \cdot 5$ & 618 & $47 \cdot 8$ & 706 & $47 \cdot 6$ & \\
\hline High school or higher & 1856 & 43.5 & 6225 & $50 \cdot 4$ & 674 & $52 \cdot 2$ & 775 & $52 \cdot 3$ & \\
\hline $\mathrm{BMI}(\mathrm{n}, \%)$ & & & & & & & & & $<0.0001$ \\
\hline Normal $(\leq 25)$ & 1216 & $28 \cdot 5$ & 3472 & $28 \cdot 1$ & 399 & $30 \cdot 9$ & 459 & $31 \cdot 0$ & \\
\hline Overweight $(>25$ and $<30)$ & 1722 & $40 \cdot 3$ & 5363 & 43.5 & 547 & $42 \cdot 3$ & 690 & $46 \cdot 6$ & \\
\hline Obese $(\geq 30)$ & 1333 & $31 \cdot 2$ & 3506 & $28 \cdot 4$ & 346 & $26 \cdot 8$ & 333 & $22 \cdot 4$ & \\
\hline Smokers (n, \%) & 1073 & $25 \cdot 1$ & 2889 & $23 \cdot 4$ & 308 & $23 \cdot 8$ & 324 & 21.9 & 0.0004 \\
\hline Leisure-time PA (n, \%) & & & & & & & & & $<0.0001$ \\
\hline$\leq 2 \cdot 27$ (below median) & 2291 & $53 \cdot 6$ & 6359 & 51.5 & 574 & $44 \cdot 4$ & 576 & $38 \cdot 9$ & \\
\hline$>2.27$ (above median) & 1980 & $46 \cdot 4$ & 5982 & $48 \cdot 5$ & 718 & $55 \cdot 6$ & 906 & $61 \cdot 1$ & \\
\hline Hypertension (n, \%) & 2380 & $55 \cdot 7$ & 6624 & $53 \cdot 7$ & 679 & $52 \cdot 6$ & 828 & $55 \cdot 9$ & 0.62 \\
\hline Systolic BP (mean and SD) & $140 \cdot 0$ & $21 \cdot 6$ & $140 \cdot 1$ & $20 \cdot 0$ & $139 \cdot 5$ & $19 \cdot 6$ & $140 \cdot 3$ & $21 \cdot 0$ & 0.58 \\
\hline Diastolic BP (mean and SD) & $82 \cdot 4$ & $9 \cdot 7$ & $82 \cdot 6$ & 9.5 & $82 \cdot 7$ & $9 \cdot 8$ & $82 \cdot 6$ & $9 \cdot 8$ & 0.58 \\
\hline Hypercholesterolaemia (n, \%) & 1235 & $28 \cdot 9$ & 3709 & $30 \cdot 1$ & 332 & $25 \cdot 7$ & 498 & $33 \cdot 6$ & $<0.0001$ \\
\hline Total cholesterol (mg/dl; mean and SD) & $210 \cdot 8$ & $42 \cdot 0$ & $215 \cdot 2$ & $41 \cdot 3$ & $210 \cdot 1$ & $41 \cdot 5$ & $217 \cdot 1$ & $39 \cdot 9$ & $<0.0001$ \\
\hline HDL-cholesterol (mg/dl; mean and SD) & $56 \cdot 4$ & $15 \cdot 0$ & $57 \cdot 6$ & $14 \cdot 8$ & $56 \cdot 6$ & $14 \cdot 6$ & $58 \cdot 5$ & $15 \cdot 0$ & $<0.0001$ \\
\hline LDL-cholesterol (mg/dl; mean and SD) & $128 \cdot 6$ & $34 \cdot 9$ & $132 \cdot 1$ & $34 \cdot 7$ & $128 \cdot 6$ & $34 \cdot 7$ & $133 \cdot 7$ & $33 \cdot 7$ & $<0.0001$ \\
\hline TAG (mg/dl; mean and SD) & 131.4 & 84.4 & $129 \cdot 6$ & $85 \cdot 2$ & $127 \cdot 1$ & $84 \cdot 8$ & $128 \cdot 1$ & $87 \cdot 2$ & 0.30 \\
\hline Diabetes (n, \%) & 467 & $10 \cdot 9$ & 942 & $7 \cdot 6$ & 92 & $7 \cdot 1$ & 128 & $8 \cdot 6$ & $<0.0001$ \\
\hline Blood glucose (mg/dl; mean and SD) & $101 \cdot 6$ & $27 \cdot 2$ & $100 \cdot 4$ & $22 \cdot 8$ & $99 \cdot 2$ & 22.9 & $100 \cdot 8$ & $22 \cdot 7$ & 0.0020 \\
\hline Energy intake (kJ/d; mean and SD) & $7932 \cdot 8$ & $2301 \cdot 2$ & $8748 \cdot 7$ & $2192 \cdot 4$ & $9250 \cdot 8$ & $2192 \cdot 4$ & 9539.5 & $2217 \cdot 5$ & $<0.0001$ \\
\hline Energy intake (kcal/d; mean and SD) & 1896 & 550 & 2091 & 524 & 2211 & 524 & 2280 & 530 & \\
\hline Mediterranean diet score (n, \%) & & & & & & & & & $<0.0001$ \\
\hline $0-3$ & 1446 & 33.9 & 3871 & 31.4 & 321 & $24 \cdot 9$ & 330 & $22 \cdot 3$ & \\
\hline $4-5$ & 1849 & $43 \cdot 3$ & 5433 & $44 \cdot 0$ & 560 & $43 \cdot 3$ & 643 & $43 \cdot 4$ & \\
\hline$>5$ & 976 & $22 \cdot 9$ & 3037 & $24 \cdot 6$ & 411 & $31 \cdot 8$ & 509 & $34 \cdot 4$ & \\
\hline \multicolumn{10}{|l|}{ Biomarkers of inflammation } \\
\hline $\mathrm{CRP}(\mathrm{mg} / \mathrm{l}) \dagger$ & 1.54 & $1.49,1.58$ & 1.48 & $1.46,1.51$ & $1 \cdot 36$ & $1.29,1.44$ & $1 \cdot 31$ & $1 \cdot 24,1 \cdot 37$ & 0.0048 \\
\hline Leucocyte count $\left(10^{9} / /\right.$ means and SD $) \dagger$ & $6 \cdot 28$ & 1.67 & $6 \cdot 22$ & $1 \cdot 72$ & $6 \cdot 14$ & 1.52 & $6 \cdot 16$ & 1.57 & 0.77 \\
\hline Platelet count $\left(10^{9} / \mathrm{I}\right.$ means and SD $) \dagger$ & $248 \cdot 1$ & $64 \cdot 1$ & $250 \cdot 6$ & $64 \cdot 4$ & $249 \cdot 4$ & $59 \cdot 1$ & 246.5 & $59 \cdot 7$ & 0.044 \\
\hline Neutrophil/lymphocyte $†$ & 2.01 & 0.79 & 1.99 & 0.82 & 1.95 & 0.78 & 1.92 & 0.70 & 0.0053 \\
\hline
\end{tabular}

PA, physical activity; BP, blood pressure; CRP, C-reactive protein.

Means for biomarkers of inflammation, biochemical parameters and systolic or diastolic BP are adjusted for age and sex. All $P$ values are adjusted for age and sex. Values for CRP are reported as geometric means with corresponding $95 \%$ confidence intervals.

* Numbers do not add up to $100 \%$ because of missing values.

$\dagger P$ values obtained from the model adjusted for age, sex, educational level (low/high), smoking (never, smoker, former), leisure-time PA (continuous), BMI (continuous), energy intake (continuous), Mediterranean diet score without nuts (continuous).

Table 2. Risk of death associated with the frequency of nut intake (Hazard ratios and $95 \%$ confidence intervals)

\begin{tabular}{|c|c|c|c|c|c|c|c|c|c|}
\hline & \multicolumn{8}{|c|}{ Frequency of nut intake } & \multirow[b]{3}{*}{$P$ trend } \\
\hline & \multicolumn{2}{|c|}{ Never } & \multicolumn{2}{|c|}{$\leq 2$ times/month } & \multicolumn{2}{|c|}{ 3-7 times/month } & \multicolumn{2}{|c|}{$\geq 8$ times/month } & \\
\hline & Hazard ratio & $95 \% \mathrm{Cl}$ & Hazard ratio & $95 \% \mathrm{Cl}$ & Hazard ratio & $95 \% \mathrm{Cl}$ & Hazard ratio & $95 \% \mathrm{Cl}$ & \\
\hline Number of deaths/number of subjects & \multicolumn{2}{|c|}{$122 / 4271$} & \multicolumn{2}{|c|}{$182 / 12341$} & \multicolumn{2}{|c|}{$13 / 1292$} & \multicolumn{2}{|c|}{$17 / 1482$} & - \\
\hline Crude & -reference- & & 0.50 & $0.40,0.63$ & 0.37 & $0.21,0.65$ & 0.40 & $0.24,0.66$ & $<0.0001$ \\
\hline Age/sex adjusted & -reference- & & 0.63 & $0.50,0.80$ & 0.49 & $0.27,0.86$ & 0.44 & $0.26,0.73$ & $<0.0001$ \\
\hline Model 1 & -reference- & & 0.68 & $0.54,0.87$ & 0.56 & $0.31,1.00$ & 0.53 & $0.32,0.90$ & 0.0010 \\
\hline Model 2 & -reference- & & 0.68 & $0.54,0.87$ & 0.57 & $0.32,1.02$ & 0.55 & $0.33,0.92$ & 0.0015 \\
\hline
\end{tabular}

Model 1: multivariable hazard ratios with corresponding $95 \%$ confidence intervals obtained from the model adjusted for age, sex, educational level (low/high), smoking (never, smoker, former), leisure-time physical activity (continuous), BMI (continuous), energy intake (continuous), Mediterranean diet score without nuts (continuous). Model 2: same as model 1, but further controlled for biomarkers of inflammation (C-reactive protein, platelet count and the neutrophil to lymphocyte ratio). 
Table 3. Cause-specific risks of death by nut intake v. no intake (Hazard ratios and $95 \%$ confidence intervals)

\begin{tabular}{|c|c|c|c|c|c|c|c|c|c|}
\hline & \multirow[b]{3}{*}{$\begin{array}{c}\text { Number of deaths/number } \\
\text { of subjects }\end{array}$} & \multicolumn{6}{|c|}{ Nut intake } & \multirow[b]{3}{*}{$P(1)$} & \multirow[b]{3}{*}{$P(2)$} \\
\hline & & \multicolumn{2}{|c|}{ Age/sex adjusted } & \multicolumn{2}{|c|}{ Model 1} & \multicolumn{2}{|c|}{ Model 2} & & \\
\hline & & $\begin{array}{l}\text { Hazard } \\
\text { ratio }\end{array}$ & $95 \% \mathrm{Cl}$ & $\begin{array}{c}\text { Hazard } \\
\text { ratio }\end{array}$ & $95 \% \mathrm{Cl}$ & $\begin{array}{l}\text { Hazard } \\
\text { ratio }\end{array}$ & $95 \% \mathrm{Cl}$ & & \\
\hline $\begin{array}{l}\text { All-cause } \\
\text { mortality }\end{array}$ & $334 / 19386$ & 0.60 & $0.48,0.75$ & 0.66 & $0.53,0.83$ & 0.66 & $0.53,0.84$ & 0.0004 & 0.0005 \\
\hline CVD mortality & $104 / 19386$ & 0.73 & $0.49,1.11$ & 0.87 & $0.57,1.32$ & 0.87 & $0.57,1.32$ & 0.50 & 0.50 \\
\hline CHD mortality & $39 / 19386$ & 0.56 & $0.29,1.08$ & 0.74 & $0.38,1.45$ & 0.76 & $0.39,1.49$ & 0.39 & 0.43 \\
\hline Stroke mortality & $19 / 19386$ & 0.93 & $0.35,2.47$ & 0.98 & $0.36,2.66$ & 1.01 & $0.37,2.76$ & 0.97 & 0.98 \\
\hline Cancer mortality & $124 / 19386$ & 0.58 & $0.40,0.85$ & 0.64 & $0.44,0.94$ & 0.64 & $0.44,0.94$ & 0.022 & 0.023 \\
\hline
\end{tabular}

Model 1 and $P(1)$ : hazard ratios with 95\% confidence intervals and $P$ values obtained from the model adjusted for age, sex, educational level (low/high), smoking (never, smoker, former), leisure-time physical activity (continuous), BMI (continuous), energy intake (continuous) and Mediterranean diet score without nuts (continuous). Model 2 and $P(2)$ : same as in model 1, but further controlled for C-reactive protein platelet count and the neutrophil to lymphocyte ratio.

Table 4. Subgroup analyses by nut intake $v$. no intake for total mortality

(Hazard ratios and $95 \%$ confidence intervals)

\begin{tabular}{|c|c|c|c|c|c|}
\hline & \multirow[b]{3}{*}{ Number of deaths/number of subjects } & \multicolumn{3}{|c|}{ Nut intake } & \multirow[b]{3}{*}{$P$ for interaction } \\
\hline & & \multicolumn{2}{|c|}{ Multivariable HR } & \multirow[b]{2}{*}{$P$ value } & \\
\hline & & $\mathrm{HR}$ & $95 \% \mathrm{Cl}$ & & \\
\hline All & $334 / 19386$ & 0.68 & $0.54,0.85$ & 0.0009 & - \\
\hline \multicolumn{6}{|l|}{ Sex } \\
\hline Women & $106 / 10509$ & 0.88 & $0.59,1.33$ & 0.54 & 0.23 \\
\hline Men & $228 / 8877$ & 0.59 & $0.44,0.77$ & 0.0002 & \\
\hline \multicolumn{6}{|l|}{ Age } \\
\hline$\leq 65$ & $109 / 15587$ & 0.83 & $0.53,1.30$ & 0.40 & 0.32 \\
\hline$>65$ & 225/3799 & 0.62 & $0.47,0.81$ & 0.0006 & \\
\hline \multicolumn{6}{|l|}{ Smoking habit } \\
\hline No & $135 / 9821$ & 0.76 & $0.53,1.10$ & 0.15 & 0.35 \\
\hline Yes & $85 / 4595$ & 0.82 & $0.51,1.32$ & 0.41 & \\
\hline Former & $114 / 4970$ & 0.50 & $0.34,0.74$ & 0.0005 & \\
\hline \multicolumn{6}{|l|}{ BMI } \\
\hline$<30$ & $221 / 13868$ & 0.68 & $0.51,0.91$ & 0.0083 & 0.92 \\
\hline$\geq 30$ & $113 / 5518$ & 0.63 & $0.42,0.93$ & 0.021 & \\
\hline \multicolumn{6}{|l|}{ Diabetes } \\
\hline No & $258 / 17757$ & 0.64 & $0.49,0.83$ & 0.0009 & 0.36 \\
\hline Yes & $76 / 1629$ & 0.80 & $0.50,1.30$ & 0.37 & \\
\hline \multicolumn{6}{|c|}{$\begin{array}{l}\text { Mediterranean diet score } \\
\text { (without nuts) }\end{array}$} \\
\hline $0-3$ & $98 / 5968$ & 0.47 & $0.31,0.71$ & 0.0003 & 0.022 \\
\hline $4-5$ & $150 / 8485$ & 0.73 & $0.52,1.04$ & 0.085 & \\
\hline$>5$ & $86 / 4933$ & 0.91 & $0.56,1.47$ & 0.69 & \\
\hline \multicolumn{6}{|c|}{ Metabolic syndrome* } \\
\hline No & $199 / 14485$ & 0.68 & $0.51,0.92$ & 0.013 & 0.86 \\
\hline Yes & $135 / 4896$ & 0.66 & $0.46,0.94$ & 0.021 & \\
\hline
\end{tabular}

Hazard ratios (HR) with $95 \%$ confidence intervals and $P$ values obtained from the model adjusted for age, sex, educational level (low/high), smoking (never, smoker, former), leisure-time physical activity (continuous), BMI (continuous), energy intake (continuous), Mediterranean diet score without nuts (continuous).

* Numbers do not add up to $100 \%$ because of missing values.

cancer death, comparable with a $36 \%$ reduction in cancer mortality observed in our study. As the PREDIMED study was conducted within high CVD risk subjects older than 55 years, our study somehow extends the positive results documented in the PREDIMED trial to a more general, younger population. The actual role of nut intake in relation to cancer risk surely deserves further investigation ${ }^{(9)}$.

When specific CVD mortality was considered, a nonstatistically significant trend for protection was found. However, when only CHD death was considered, the reduction in death risk was similar to that observed for cancer mortality, but, most probably, the small number of CHD cases did not allow to reach any statistical significance. Yet, a recent meta-analysis found that cardiovascular advantages from nut consumption were primarily driven by decreased coronary artery disease deaths ${ }^{(34)}$.

It is noteworthy that the association of nut consumption with stroke deaths was weaker than that observed for other causes, and no statistical significance was reached. Our overall results on CVD events also appear in agreement with data from 
a meta-analysis that suggested similar protection for CVD, ischaemic heart disease and total mortality, but no protection for type 2 diabetes or stroke ${ }^{(7)}$.

However, benefits from nut intake against diabetes were observed in the PREDIMED-Reus trial ${ }^{(35)}$.

\section{Subgroup analyses}

Our study has also examined the effects of nut intake within subgroups at different risk for both CVD or cancer. The protection against mortality offered by nut intake was more evident in subjects with poor adherence to the MD as compared with those showing greater adherence. Nut intake appeared to add no additional protection against mortality within individuals who already followed an MD, probably because they already benefit from other fundamental components of this healthy dietary pattern, such as olive oil, moderate alcohol intake or antioxidantrich foods. Conversely, nut intake alone seems to convey some health advantage to those who stick less to this dietary pattern. These data are in agreement with those observed in nonMediterranean population settings, such as in the USA, in which health advantages from nut consumption have been reported ${ }^{(36)}$.

\section{Role of inflammation}

Finally, we addressed the possible role of low-grade inflammation on the causal pathway between nuts and the risk of death. Results showed that subjects consuming higher amounts of nuts had lower values of biomarkers of inflammation (CRP, platelet count or NLR), probably indicating a reduced subclinical inflammatory status in nut consumers, in agreement with previous studies ${ }^{(5)}$. However, we observed that inflammation did not account for the inverse associations between nut intake and overall or cancer deaths. Similarly, adjustment for lipid status did not modify the association.

\section{Strengths and limitations of this study}

The strengths of this large epidemiological study in a Mediterranean population are represented by its prospective nature. In addition, further control of all analyses by a wide panel of possible confounding factors and diet-related behaviours should assure consistency to the observed association.

A major limitation of the present study in an apparently healthy population is the relatively small number of deaths, but this did not prevent statistically significant differences to be consistently observed, at least for both total and cancer mortality. The relatively low number of CVD, CHD or stroke events did not allow to provide conclusive and consistent results or to explore more deeply the plateau effect, which may be linked to specific death causes (i.e. stroke, for which a J-shape was already observed ${ }^{(37)}$ ) rather than others. As far as total and cancer deaths are concerned, our study did reach the same major conclusions of a much larger population study ${ }^{(18)}$.

\section{Conclusions}

The present results obtained in people living in a Mediterranean country provide evidence of an inverse association between nut consumption and risk of death in a general population that is apparently free from CVD and cancer at enrolment. To our knowledge, this study is one of the first observational investigations to address the health advantages of nut intake for subjects at risk for CVD or cancer, such as subjects who are obese, affected by diabetes, metabolic syndrome or are less adherent to the MD. In addition, this study adds knowledge on the relationship between nut consumption and cancer, an issue on which evidence is still scarce and controversial.

Finally, our findings suggest the opportunity to consider nuts as an important part of a healthy dietary pattern for the prevention of chronic diseases, as already suggested by the PREDIMED study ${ }^{(8,19,38,39)}$. Possibly, nut consumption should be mainly encouraged in countries in which nut intake is not regular and MD is not followed as a regular eating pattern.

The health advantages of nuts observed at low to moderate doses are in agreement with the beneficial effects of specific foods such as salt ${ }^{(40)}$, dark chocolate ${ }^{(41)}$ or alcohol ${ }^{(42)}$, whose protective role has only been detected at low to moderate intakes.

\section{Acknowledgements}

The Moli-sani research group thanks Dr Vittorio Krogh and Dr Sabina Sieri (Istituto Nazionale dei Tumori, Milan, Italy) for their contribution to dietary questionnaire analysis and interpretation, the Associazione Cuore Sano Onlus (Campobasso) for its financial support and the Azienda Sanitaria Regionale del Molise (ASReM, Campobasso, Italy), the Offices of vital statistics of the Molise region and the Molise Dati Spa (Campobasso, Italy) for their collaboration and support provided during the follow-up activities.

The enrolment phase of the Moli-sani Project was conducted at the Research Laboratories of the Catholic University in Campobasso (Italy) and supported by research grants from Pfizer Foundation (Rome, Italy), the Italian Ministry of University and Research (MIUR, Rome, Italy) - Programma Triennale di Ricerca, Decreto no. 1588 and Instrumentation Laboratory, Milan, Italy. These funders had no role in study design, collection, analysis and interpretation of data; in the writing of the report; and in the decision to submit the article for publication. All authors were and are independent from funders.

M. B., L. I. and A. Di. C. designed the present research; A. De. C., S. C., M. P. and F. B. managed data collection; M. B. and A. Di. C. analysed the data; M. B. wrote the paper; and M. B. D., G. d. G. and L. I. originally inspired the research and critically reviewed the manuscript.

There are no conflicts of interest.

Marialaura Bonaccio was supported by a Fondazione Umberto Veronesi Fellowship.

\section{References}

1. Sofi F, Abbate R, Gensini GF, et al. (2010) Accruing evidence on benefits of adherence to the Mediterranean diet on health: an updated systematic review and meta-analysis. Am J Clin Nutr 92, 1189-1196.

2. Bonaccio M, Iacoviello L \& de Gaetano G (2012) The Mediterranean diet: the reasons for a success. Thromb Res 129, 401-404. 
3. Estruch R (2010) Anti-inflammatory effects of the Mediterranean diet: the experience of the PREDIMED study. Proc Nutr Soc 69, 333-340.

4. Ros E, Tapsell LC \& Sabaté J (2010) Nuts and berries for heart health. Curr Atheroscler Rep 12, 397-406.

5. Jiang R, Jacobs DR Jr, Mayer-Davis E, et al. (2006) Nut and seed consumption and inflammatory markers in the multiethnic study of atherosclerosis. Am J Epidemiol 163, 222-231.

6. Zhou D, Yu H, He F, et al. (2014) Nut consumption in relation to cardiovascular risk and type 2 diabetes: a systematic review and meta-analysis of prospective studies. Am J Clin Nutr 100, 270-277.

7. Luo C, Zhang Y, Ding Y, et al. (2014) Nut consumption and risk of type 2 diabetes, cardiovascular disease, and all-cause mortality: a systematic review and meta-analysis. Am J Clin Nutr 100, 256-269.

8. Estruch R, Ros E, Salas-Salvadó J, et al. (2013) Primary prevention of cardiovascular disease with a Mediterranean diet. N Engl J Med 368, 1279-1290.

9. Sabaté J \& Ang Y (2009) Nuts and health outcomes: new epidemiologic evidence. Am J Clin Nutr 89, 1643S-1648S.

10. Kris-Etherton PM, Hu FB, Ros E, et al. (2008) The role of tree nuts and peanuts in the prevention of coronary heart disease: multiple potential mechanisms. J Nutr 138, 1746S-1751S.

11. Steffen LM, Kroenke CH, Yu X, et al. (2005) Associations of plant food, dairy product, and meat intakes with 15-y incidence of elevated blood pressure in young black and white adults: the Coronary Artery Risk Development in Young Adults (CARDIA) Study. Am J Clin Nutr 82, 1169-1177.

12. Doménech M, Roman P, Lapetra J, et al. (2014) Mediterranean diet reduces 24-hour ambulatory blood pressure, blood glucose, and lipids: one-year randomized, clinical trial. Hypertension 64, 69-76.

13. Mukuddem-Petersen J, Oosthuizen W \& Jerling JC (2005) A systematic review of the effects of nuts on blood lipid profiles in humans. J Nutr 135, 2082-2089.

14. Sabaté J, Oda K \& Ros E (2010) Nut consumption and blood lipid levels: a pooled analysis of 25 intervention trials. Arch Intern Med 170, 821-827.

15. Jiang R, Manson JE, Stampfer MJ, et al. (2002) Nut and peanut butter consumption and risk of type 2 diabetes in women. JAMA 288, 2554-2560.

16. Afshin A, Micha R, Khatibzadeh S, et al. (2014) Consumption of nuts and legumes and risk of incident ischemic heart disease, stroke, and diabetes: a systematic review and metaanalysis. Am J Clin Nutr 100, 278-288.

17. Salas-Salvadó J, Guasch-Ferré M, Bulló M, et al. (2014) Nuts in the prevention and treatment of metabolic syndrome. Am J Clin Nutr 100, 399S-407S.

18. Bao Y, Han J, Hu FB, et al. (2013) Association of nut consumption with total and cause-specific mortality. N Engl J Med 369, 2001-2011.

19. Guasch-Ferré M, Bulló M, Martínez-González MÁ, et al. (2013) Frequency of nut consumption and mortality risk in the PREDIMED nutrition intervention trial. BMC Med 11, 164.

20. Albert CM, Gaziano JM, Willett WC, et al. (2002) Nut consumption and decreased risk of sudden cardiac death in the Physicians' Health Study. Arch Intern Med 162, 1382-1387.

21. Fernández-Montero A, Bes-Rastrollo M, Barrio-López MT, et al. (2014) Nut consumption and 5-y all-cause mortality in a Mediterranean cohort: the SUN project. Nutrition 30, 1022-1027.

22. Bonaccio M, Di Castelnuovo A, De Curtis A, et al. (2014) Adherence to the Mediterranean diet is associated with lower platelet and leukocyte counts: results from the Moli-sani study. Blood 123, 3037-3044.

23. Iacoviello L, Bonanni A, Costanzo S, et al. (2007) The Molisani Project, a randomized, prospective cohort study in the
Molise region in Italy; design, rationale and objectives. Ital J Public Health 4, 110-118.

24. Pala V, Sieri S, Palli D, et al. (2003) Diet in the Italian EPIC cohorts: presentation of data and methodological issues. Tumori 89, 594-607.

25. Pisani P, Faggiano F, Krogh V, et al. (1997) Relative validity and reproducibility of a food frequency dietary questionnaire for use in the Italian EPIC centres. Int J Epidemiol 26, Suppl. 1, S152-S160.

26. Trichopoulou A, Costacou T, Bamia C, et al. (2003) Adherence to a Mediterranean diet and survival in a Greek population. N Engl J Med 348, 2599-2608.

27. Cleeman JI (2001) Executive summary of the third report of the National Cholesterol Education Program (NCEP) expert panel on detection, evaluation, and treatment of high blood cholesterol in adults (adult treatment panel III). J Am Med Assoc 285, 2486-2497.

28. Bhat T, Teli S, Rijal J, et al. (2013) Neutrophil to lymphocyte ratio and cardiovascular diseases: a review. Expert Rev Cardiovasc Ther 11, 55-59.

29. Grundy SM, Cleeman JI, Daniels SR, et al. (2005) Diagnosis and management of the metabolic syndrome: an American Heart Association/National Heart, Lung, and Blood Institute Scientific Statement. Circulation 112, 2735-2752.

30. Pereira MA, Fitzgerald SJ, Gregg EW, et al. (1997) A collection of physical activity questionnaires for health-related research: The Monica Optional Study of Physical Activity (MOSPA). Med Sci Sports Exerc 29, S162-S169.

31. Jenab M, Ferrari P, Slimani N, et al. (2004) Association of nut and seed intake with colorectal cancer risk in the European Prospective Investigation into Cancer and Nutrition. Cancer Epidemiol Biomarkers Prev 13, 1595-1603.

32. Petridou E, Kedikoglou S, Koukoulomatis P, et al. (2002) Diet in relation to endometrial cancer risk: a case control study in Greece. Nutr Cancer 44, 16-22.

33. Gonzalez CA \& Salas-Salvado J (2006) The potential of nuts in the prevention of cancer. Br J Nutr 96, S87-S94.

34. Grosso G, Yang J, Marventano S, et al. (2015) Nut consumption on all-cause, cardiovascular, and cancer mortality risk: a systematic review and meta-analysis of epidemiologic studies. Am J Clin Nutr 101, 783-793.

35. Salas-Salvadó J, Bulló M, Babio N, et al. (2011) Reduction in the incidence of type 2 diabetes with the Mediterranean diet: results of the PREDIMED-Reus nutrition intervention randomized trial. Diabetes Care 34, 14-19.

36. Rohrmann S \& Faeh D (2013) Should we go nuts about nuts? BMC Med 11, 165.

37. Djoussé L, Gaziano JM, Kase CS, et al. (2010) Nut consumption and risk of stroke in US male physicians. Clin Nutr 29, 605-609.

38. Babio N, Toledo E, Estruch R, et al. (2014) Mediterranean diets and metabolic syndrome status in the PREDIMED randomized trial. CMAJ 186, E649-E657.

39. Salas-Salvadó J, Bulló M, Estruch R, et al. (2014) Prevention of diabetes with Mediterranean diets: a subgroup analysis of a randomized trial. Ann Intern Med 160, 1-10.

40. O'Donnell M, Mente A, Rangarajan S, et al. (2014) Urinary sodium and potassium excretion, mortality, and cardiovascular events. $N$ Engl J Med 371, 612-623.

41. di Giuseppe R, Di Castelnuovo A, Centritto F, et al. (2008) Regular consumption of dark chocolate is associated with low serum concentrations of C-reactive protein in a healthy Italian population. J Nutr 138, 1939-1945.

42. Di Castelnuovo A, Costanzo S, Bagnardi V, et al. (2006) Alcohol dosing and total mortality in men and women: an updated meta-analysis of 34 prospective studies. Arch Intern Med 166, 2437-2445. 


\section{Appendix: Moli-sani study investigators}

\section{Steering committee}

Licia Iacoviello (Neuromed, Pozzilli, Italy), Chairperson, Maria Benedetta Donati and Giovanni de Gaetano (Neuromed, Pozzilli, Italy) and Simona Giampaoli (Istituto Superiore di Sanità, Roma, Italy).

\section{Safety and data monitoring committee}

Jos Vermylen (Catholic Univesity, Leuven, Belgio), Chairman, Ignacio De Paula Carrasco (Accademia Pontificia Pro Vita, Roma, Italy), Fabrizio Oleari (Istituto Superiore di Sanità, Roma, Italy) and Antonio Spagnolo (Catholic University, Roma, Italy).

\section{Event adjudicating committee}

Deodato Assanelli (Brescia, Italy), Vincenzo Centritto (Campobasso, Italy), Paola Muti (Hamilton, Ontario, Canada), Holger Schünemann (Hamilton, Ontario, Canada), Pasquale Spagnuolo and Dante Staniscia (Termoli, Italy).

\section{Scientific and organizing secretariat}

Francesco Zito (Coordinator), Americo Bonanni, Chiara Cerletti, Amalia De Curtis, Augusto Di Castelnuovo, Licia Iacoviello, Roberto Lorenzet, Antonio Mascioli, Marco Olivieri and Domenico Rotilio.

\section{Data management and analysis}

Augusto Di Castelnuovo (Coordinator), Marialaura Bonaccio, Simona Costanzo and Francesco Gianfagna.

\section{Informatics}

Marco Olivieri (Coordinator), Maurizio Giacci, Antonella Padulo and Dario Petraroia.

\section{Biobank and biochemical analyses}

Amalia De Curtis (Coordinator), Federico Marracino, Maria Spinelli and Christian Silvestri.

\section{Communication and press office}

Americo Bonanni (Coordinator), Marialaura Bonaccio and Francesca De Lucia.

\section{Moli-family project}

Francesco Gianfagna and Branislav Vohnout.

\section{Recruitment staff}

Franco Zito (General Coordinator); Secretariat: Mariarosaria Persichillo (Coordinator), Angelita Verna, Maura Di Lillo, Irene Di Stefano; Blood sample: Agostino Pannichella, Antonio Rinaldo Vizzarri, Branislav Vohnout, Agnieszka Pampuch; Spirometry: Antonella Arcari (Coordinator), Daniela Barbato, Francesca Bracone, Simona Costanzo, Carmine Di Giorgio, Sara Magnacca, Simona Panebianco, Antonello Chiovitti, Federico Marracino, Sergio Caccamo, Vanesa Caruso; Electrocardiograms: Livia Rago (Coordinator), Daniela Cugino, Francesco Zito, Francesco Gianfagna, Alessandra Ferri, Concetta Castaldi, Marcella Mignogna; Tomasz Guszcz; Questionnaires: Romina di Giuseppe (Coordinator), Paola Barisciano, Lorena Buonaccorsi, Floriana Centritto, Antonella Cutrone, Francesca De Lucia, Francesca Fanelli, Iolanda Santimone, Anna Sciarretta, Maura Di Lillo, Isabella Sorella, Irene Di Stefano, Emanuela Plescia, Alessandra Molinaro and Christiana Cavone.

Call center

Giovanna Galuppo, Maura Di Lillo, Concetta Castaldi, Dolores D'Angelo and Rosanna Ramacciato. 